\title{
CONNECT - Uma medida de continuidade do cuidado em serviços de saúde mental: adaptação transcultural e confiabilidade da escala
}

\author{
CONNECT - A measure of continuity of care in mental health \\ services: cross-cultural adaptation and reliability of the scale \\ Michelli Melo Grama', Diego de Lima Fonseca', Giovanni Marcos Lovisi², Lúcia Abelha Lima³
}

\section{RESUMO}

Objetivo: Realizar estudos de evidências quanto à adequação transcultural e a confiabilidade da versão em português (Brasil) da CONNECT - Uma medida de continuidade do cuidado em serviços de saúde mental, em uma amostra de 50 indivíduos com transtornos mentais graves. Métodos: A adaptação transcultural foi realizada segundo o processo de tradução e adaptação de instrumentos (WHO). A confiabilidade foi avaliada pela consistência interna por meio do coeficiente alfa de Cronbach e pela estabilidade ao longo do tempo por meio do kappa ponderado, kappa ajustado pela prevalência (PABAK) e coeficiente de correlação intraclasse (ICC). Resultados: $O$ processo de tradução e retrotradução da CONNECT resultou na versão em português adaptada para a cultura brasileira. Na apreciação da confiabilidade pelo PABAK, 34 itens apresentaram confiabilidade moderada, importante ou quase perfeita. A concordância pelo ICC apresentou-se fraca ou pobre para três subescalas, mas a maioria dos itens apresentou percentual de concordância acima de 50\%. A consistência interna pelo alfa de Cronbach foi de moderada a alta, para três subescalas e para a composição da escala total, denotando a adequação e a coerência dos itens. Conclusão: A CONNECT é a primeira medida de continuidade do cuidado adaptada para contexto cultural brasileiro. Sua adaptação mostrou adequação satisfatória entre a versão original e a versão em português.

\section{Palavras-chave}

Adaptação transcultural, confiabilidade, CONNECT, continuidade da assistência ao paciente, serviços de saúde mental.
Os resultados para a maioria das subescalas se mostraram satisfatórios, porém diferiram de outros estudos sobre a CONNECT. Este estudo constitui a primeira fase para a obtenção de uma medida de continuidade do cuidado válida para pacientes brasileiros com transtornos mentais graves e persistentes, e estudos adicionais para examinar a validade ainda se fazem necessários.

\section{ABSTRACT}

Objective: Perform evidence studies and cross-cultural adaptation and the reliability of the Portuguese version (Brazil) of the CONNECT - A measure of continuity of care in mental health services, in a sample of 50 individuals with severe mental illnesses. Methods: The

1 Universidade Federal do Rio de Janeiro (UFRJ), Instituto de Estudos em Saúde Coletiva.

2 Universidade de Londres, Instituto de Psiquiatria; UFRJ, Instituto de Estudos em Saúde Coletiva.

3 Fundação Oswaldo Cruz; UFRJ, Instituto de Estudos em Saúde Coletiva.

Recebido em

$11 / 9 / 2015$

Aprovado em

$28 / 3 / 2016$

DOI: 10.1590/0047-2085000000115

\author{
Endereço para correspondência: Michelli Melo Grama \\ Instituto de Estudos em Saúde Coletiva, \\ Universidade Federal do Rio de Janeiro \\ Praça Jorge Machado Moreira, Ilha do Fundão, Cidade Universitária \\ 21944-970 - Rio de Janeiro, RJ, Brasil \\ Telefone: (21) 98803-1163 \\ E-mail: michellimelo@oi.com.br
}




\section{Keywords}

Cross-cultural adaptation, reliability, CONNECT, continuity of patient care, mental health services. cross-cultural adaptation was performed according to the process of translation and adaptation of instruments (WHO). Reliability was assessed by internal consistency by Cronbach's alpha coefficient and by stability over time through the weighted kappa, kappa adjusted for prevalence (PABAK) and intraclass correlation coefficient (ICC). Results: The process of translation and back translation of CONNECT resulted in the Portuguese version adapted to Brazilian culture. In the assessment of reliability for PABAK, 34 items showed moderate reliability and significant or almost perfect. The agreement by the ICC was weak or poor for three subscales, but since most of the items presented agreement percentage above $50 \%$. The internal consistency by Cronbach's alpha was moderate to high for three subscales and to the composition of full scalle denoting the appropriateness and consistency of the items.

Conclusion: CONNECT is the first measure of continuity of care adapted to the Brazilian cultural context. Its adaptation showed satisfactory suitability of equivalence between the original version and the version in Portuguese. The results for most subscales were satisfactory, however, differed from other studies on the CONNECT. This study is the first phase to obtain a valid measure of continuity of care for Brazilian patients with severe mental illnesses and additional studies to examine the validity of the CONNECT still are needed.

\section{INTRODUÇÃO}

O tema continuidade do cuidado em saúde mental tem sido amplamente discutido nas literaturas internacionais e, mais recentemente, nas nacionais, principalmente por causa dos avanços no processo de desinstitucionalização, da implantação da rede de atenção psicossocial e da necessidade de se consolidar a reforma psiquiátrica no país'.

Bachrach, que elaborou artigo central sobre o tema, define continuidade do cuidado em saúde mental como o fluxo ordenado e ininterrupto dos pacientes entre os diversos elementos do sistema de prestação de serviço. Segundo o autor, para que haja continuidade do cuidado, esta deve se iniciar tão logo o paciente entra no serviço de saúde, seja no nível primário, secundário ou terciário, e terminar somente quando as necessidades do paciente cessarem².

Nesse contexto, a continuidade do cuidado tem sido considerada essencial para o planejamento de ações e serviços, principalmente para os indivíduos com transtorno mental grave que necessitam de longos tratamentos em diferentes serviços, e também para fornecer uma integração entre os serviços de saúde mental e outros serviços, seja de saúde ou não².

O reconhecimento da importância da continuidade do cuidado tem levado ao desenvolvimento crescente de instrumentos de medida para diferentes grupos de pacientes. Em 2003, nos Estados Unidos, Ware et al. ${ }^{3}$ desenvolveram uma medida formal de continuidade do cuidado que reflete as perspectivas dos usuários dos serviços de saúde mental, a CONNECT, que é uma ferramenta de avaliação da continuidade do cuidado em serviços de saúde mental, designada para pacientes com transtornos mentais graves e que serve como um indicador da qualidade do atendimento nesses serviços. Ela mede a interação/relacionamento entre pro- fissionais e usuários dos serviços de saúde mental, considerando as atividades diárias de fornecimento e recebimento de cuidados.

A aplicação da CONNECT é realizada por meio de aplicação de questionário pelo entrevistador aos pacientes, utilizando um questionário de 59 questões com respostas fixas que pontuam de 1 a 5 segundo o método Likert de soma de pontuações, que varia de 59 a 295 pontos (quanto maior o escore, melhor a continuidade do cuidado).

Em 2007, a CONNECT foi traduzida e adaptada para o espanhol por Chavez et al. ${ }^{4}$ e apresentou resultados semelhantes aos da versão original.

No Brasil, embora a importância da continuidade do cuidado para os pacientes com transtornos mentais graves seja reconhecida, ainda não dispomos de um instrumento adequadamente validado para mensurá-la. Desse modo, fica evidente a necessidade de um instrumento que seja capaz de contemplar todas as dimensões da continuidade do cuidado, adaptado para a realidade brasileira, com escalas que possuam propriedades psicométricas estáveis, com boa confiabilidade e com resultados que direcionem ações e práticas de cuidado, já que falhas na continuidade afetam negativamente o curso e o prognóstico dos pacientes com transtornos mentais graves ${ }^{5}$.

Dessa maneira, a CONNECT pode orientar o acompanhamento dos pacientes com transtornos mentais graves e o planejamento e desenvolvimento de ações que aumentem a resolutividade dos serviços, contribuindo, assim, para os avanços da reforma psiquiátrica.

Com isso, este estudo objetivou realizar a adaptação transcultural da CONNECT - uma medida de continuidade de cuidados em serviços de saúde mental para a população brasileira com transtornos mentais graves e testar suas propriedades psicométricas em relação à confiabilidade. 


\section{MÉTODOS}

As etapas de adaptação transcultural da CONNECT para o contexto cultural brasileiro foram realizadas segundo o processo de tradução e adaptação de instrumentos - World Health Organization $(\mathrm{WHO})^{6}$-, que tem como foco a equivalência conceitual e cultural, e não a equivalência linguística e literal. O estudo baseou-se também em literaturas nacionais e internacionais para a adequada adaptação transcultural de instrumentos de aferição $0^{7,8}$.

A versão original em inglês foi traduzida para o português por uma psiquiatra bilíngue que tem o português como língua nativa, sendo posteriormente realizado um painel de especialistas em fevereiro de 2012 em Nova York, onde foi discutida a equivalência conceitual e de itens. A retrotradução da versão em português da CONNECT para o inglês foi feita de forma independente por um tradutor profissional, que tem como língua nativa o inglês.

Para a avaliação do significado referencial, que corresponde à concordância em termos de tradução literal entre as palavras, foi utilizada a Visual Analogue Scale (VAS) ${ }^{9}$, em que a equivalência entre os pares é verificada de forma contínua, de 0 a 100\%. E para a avaliação do significado geral, que corresponde ao impacto de cada palavra no contexto cultural da população-alvo, utilizou-se uma qualificação em quatro níveis: inalterado, pouco alterado, muito alterado e completamente alterado ${ }^{10}$.

A versão preliminar para o pré-teste foi aplicada, no mês de agosto de 2013, em 10 pacientes maiores de 18 anos, com transtornos mentais graves e persistentes de acordo com a Classificação Estatística Internacional de Doenças e de Problemas Relacionados à Saúde, 10a Revisão (CID-10) ${ }^{11}$, que frequentam o Centro de Atenção Psicossocial I (CAPS I) do município de Guapimirim, estado do Rio de Janeiro. Tanto os pacientes quanto o local foram selecionados por conveniência.

A seleção dos pacientes foi baseada no público-alvo da escala, dispensando-se a aleatoriedade pelo objetivo de o pré-teste abrir mão de tais requisitos. As entrevistas desses 10 pacientes não foram utilizadas nos resultados do estudo teste-reteste realizado em seguida.

O estudo de adaptação e confiabilidade incluindo o teste-reteste foi realizado no CAPS I do município de Guapimirim, estado do Rio de Janeiro, e no Hospital-Dia do Instituto de Psiquiatria da Universidade Federal do Rio de Janeiro (IPUB/ UFRJ), entre os meses de agosto de 2013 e janeiro de 2014.

Foi realizada uma amostragem não probabilística intencional ou por julgamento, tendo-se em vista que em estudos de adaptação de escala não é necessário garantir aleatoriedade, pois o que está sendo medido não são os entrevistados, e sim o próprio instrumento, sendo, nesses casos, mais importante garantir que os resultados sejam obtidos entre aqueles aos quais a escala se aplica. Foi solicitado que as equipes técnicas do CAPS I e do Hospital-Dia indicassem para o estudo usuários maiores de 18 anos que se adequassem a qualquer uma das categorias diagnósticas de transtorno mental grave de acordo com a CID-1011 e que estivessem em tratamento há pelo menos seis meses seguidos no local de estudo. Foram utilizados como critérios de exclusão para o estudo pacientes que se recusassem a responder à entrevista e a assinar o termo de consentimento livre e esclarecido e pacientes com incapacidade de responder às perguntas. Dessa forma, foi obtida uma amostra de 50 participantes. A escolha do tamanho da amostra se baseou nos estudos de confiabilidade realizados por Bandeira et al. ${ }^{12}$ e Schlithler et al. ${ }^{13}$.

Os dados sociodemográficos foram coletados por meio de um questionário estruturado e incluíram as variáveis idade, sexo, estado civil e nível educacional, e as categorias diagnósticas foram fornecidas pelas equipes técnicas dos locais de estudo.

As entrevistas foram realizadas individualmente, em local privado e durante o horário de funcionamento dos serviços. Foram realizadas duas entrevistas com cada participante, com duração aproximada de 20 minutos e intervalo de 10 a 20 dias entre elas. Na primeira entrevista, foram aplicados um questionário sociodemográfico e a escala CONNECT, e na segunda entrevista, somente a escala CONNECT, para avaliação da confiabilidade.

O banco de dados foi criado em uma máscara de entrada no programa Epi-Info 3.5.1 e foi analisado nos pacotes estatísticos Statistical Package for the Social Sciences (SPSS) $16.0 \mathrm{e}$ PEPI-for-Windows (WinPepi).

Foram realizadas estatísticas descritivas em relação às variáveis sociodemográficas e às categorias diagnósticas.

Para avaliação da confiabilidade temporal ou intraobservador, foi utilizado o kappa ponderado (Kp) e o PABAK (Prevalence and Bias Ajusted Kappa). E para suas interpretações, utilizou-se a classificação proposta por Landis e Koch apud Sim e Wright ${ }^{14}$,em que a concordância é classificada como: pobre ( $k$ $\leq 0)$, pequena $(k=0,1-0,20)$, razoável $(k=0,21-0,40)$, moderada $(k=0,41-0,60)$, importante $(k=0,61-0,80)$ e quase perfeita $(k=0,81-1,0)$.

A concordância também foi determinada pelo coeficiente de correlação intraclasse (ICC), com intervalo de confiança de $95 \%$, calculado entre os escores de cada subescala e o escore total da CONNECT. Para interpretação, foram adotados os valores sugeridos por McDowell ${ }^{15}$, sendo: fraca ou pobre concordância ( $\leq 40)$, moderada concordância (41-60), boa ou substancial concordância (61-80) e muito boa ou quase perfeita concordância (> 81).

A consistência interna da CONNECT foi avaliada para todos os itens da escala por meio do coeficiente alfa de Cronbach, utilizando os dados da primeira e da segunda aplicação do instrumento separadamente. Sua interpretação foi feita por meio da classificação: muito baixa $(a \leq 0,30)$, baixa (a 0,30 - $\leq 0,60)$, moderada (a 0,60 - $\leq 0,75)$, alta (a 0,75 $\leq 0,90)$ e muito alta $(a>0,90)^{16}$. 
O presente estudo foi aprovado pelo Comitê de Ética em Pesquisa do Instituto de Estudos em Saúde Coletiva, Parecer no 325.988, seguindo as orientações da Resolução no 196, e não há conflitos de interesse a declarar.

\section{RESULTADOS}

A amostra do estudo foi composta por sete pacientes que frequentam o CAPS I e 43 pacientes que frequentam o Hospital-Dia. Os pacientes entrevistados tinham idade entre 23 e 60 anos, média de 46,80 anos ( $D P=9,2$ ), sendo a maioria de homens (62\%), solteiros (70\%), com menos de 12 anos de estudo (62\%), e a categoria diagnóstica mais frequente foi a esquizofrenia (68\%).

Na adaptação transcultural da CONNECT, com relação à equivalência conceitual e de itens, nenhum item foi excluído da escala original. Foi conduzido todo o processo de tradução e retrotradução da escala, incluindo os títulos das subescalas e as frases que davam início a algumas perguntas ou afirmativas. Os títulos das subescalas sofreram algumas alterações para se adequarem aos termos mais utilizados nos serviços de saúde mental brasileiros. A estrutura final da escala, com todas as subescalas e itens que a compõem, pode ser observada no Anexo 1.

$\mathrm{Na}$ avaliação do significado referencial, 49 itens (83\%) apresentaram equivalência igual ou superior a $80 \%$. E no significado geral todos os 59 itens (100\%) foram considerados como pouco alterados ou inalterados (Quadro 1).

Quadro 1. Avaliação do significado referencial e geral

\begin{tabular}{lcc}
\hline Significado referencial & & \\
\hline & $N^{\circ}$ de itens & Percentual \\
$\geq 80 \%$ & 49 & $83 \%$ \\
$60 \%-79 \%$ & 10 & $17 \%$ \\
$40 \%-59 \%$ & 0 & -- \\
$<40 \%$ & 0 & -- \\
\hline Significado geral & & \\
\hline & $N^{\circ}$ de itens & Percentual \\
Inalterado & 45 & $76,3 \%$ \\
Pouco alterado & 12 & $20,3 \%$ \\
Muito alterado & 2 & $3,4 \%$ \\
Completamente alterado & 0 & -- \\
\hline
\end{tabular}

Todas as inconsistências percebidas após análise da equivalência semântica foram discutidas e 32 itens foram adaptados. Na maioria dos itens adaptados, as estruturas das frases foram modificadas para que as perguntas e/ou afirmativas ficassem mais adequadas ao contexto cultural brasileiro e para que o português se tornasse mais compreensível ao público-alvo. Alguns itens sofreram apenas pequenas modi- ficações em suas estruturas e algumas afirmativas sofreram modificações no sentido de torná-las mais objetivas. O layout e o formato da escala também sofreram algumas modificações.

Após cumprimento de todas as etapas da adaptação transcultural, incluindo o pré-teste, foram realizadas as modificações que se fizeram necessárias, gerando, assim, a versão final em português (Brasil) da CONNECT (Anexo 1).

Na avaliação da confiabilidade, observou-se que 31 itens da escala apresentaram confiabilidade razoável, moderada e importante. Um item apresentou confiabilidade quase perfeita, dois, confiabilidade pobre e três, confiabilidade pequena. Os valores do PABAK foram superiores ao do kappa. Trinta e quatro itens apresentaram confiabilidade quase perfeita, importante e moderada e dois, confiabilidade razoável. Dois itens apresentaram grande variação quando se comparou o kappa com o PABAK (Quadro 2).

Quadro 2. Confiabilidade da CONNECT pelo coeficiente kappa ponderado e PABAK de acordo com a classificação proposta por Landis e Koch (1977 apud Sim e Wright, 2005)

\begin{tabular}{|c|c|c|c|c|c|}
\hline \multirow{2}{*}{ Valores } & \multirow{2}{*}{ Classificação } & \multicolumn{2}{|c|}{ Kappa ponderado } & \multicolumn{2}{|c|}{ PABAK } \\
\hline & & $N^{0}$ de itens & Percentual & $N^{\circ}$ de itens & Percentual \\
\hline$K=0,81-1,0$ & Quase perfeita & 1 & $1,7 \%$ & 5 & $8,5 \%$ \\
\hline$K=0,61-0,80$ & Importante & 4 & $6,8 \%$ & 19 & $32,2 \%$ \\
\hline$K=0,41-0,60$ & Moderada & 15 & $25,4 \%$ & 10 & $16,9 \%$ \\
\hline$K=0,21-0,40$ & Razoável & 11 & $18,6 \%$ & 2 & $3,4 \%$ \\
\hline$K=0,1-0,20$ & Pequena & 3 & $5,1 \%$ & 0 & -- \\
\hline$K \leq 0$ & Pobre & 2 & $3,4 \%$ & 0 & -- \\
\hline Sem resposta & -- & 23 & $39 \%$ & 23 & $39 \%$ \\
\hline
\end{tabular}

Em relação às subescalas de transição e médico clínico, somente foi possível determinar a confiabilidade pelo método estatístico kappa ponderado e PABAK para um item de uma subescala, provavelmente pela não variabilidade das respostas e pelo pequeno número de respondentes e inconsistência dos dados, respectivamente.

Quanto ao percentual de concordância, 28 itens apresentaram concordância acima de 50\%, sendo dois com $98 \%$. Quatorze itens apresentaram concordância entre 40\% e 50\%, quatro itens, entre $30 \%$ e $40 \%$ e sete itens, de $100 \%$. Para a subescala mudança de técnico de referência, não foi possível calcular o percentual de concordância pelos mesmos motivos relatados para o kappa e PABAK.

Para seis subescalas, o ICC demonstrou concordância de moderada a boa e para três subescalas, fraca ou pobre. Para três subescalas, o ICC apresentou valor igual a zero, o que indica ausência de concordância. As justificativas prováveis são as mesmas relatadas para o kappa e PABAK. A concordância para o escore total da CONNECT foi classificada como boa ou substancial, com ICC de 0,77 (Tabela 1). 
Tabela 1. Concordância para os escores das subescalas e para o escore total da CONNECT: ICC

\begin{tabular}{lcc}
\hline Subescalas & N$^{0}$ de itens & ICC(IC95\%) \\
\hline Conhecimento do Psiquiatra & 6 & $0,75(0,60-0,85)$ \\
Conhecimento do Técnico de Referência & 6 & $0,78(0,64-0,87)$ \\
$\begin{array}{l}\text { Disponibilidade do Psiquiatra } \\
\text { Disponibilidade do Técnico de Referência }\end{array}$ & 3 & $0,54(0,31-0,71)$ \\
$\begin{array}{l}\text { Psiquiatra/Técnico de Referência/ } \\
\text { Profissional do CAPS }\end{array}$ & 7 & $0,41(0,15-0,62)$ \\
$\begin{array}{l}\text { Flexibilidade do Psiquiatra/Técnico de } \\
\text { Referência }\end{array}$ & 6 & $0,39(0,12-0,60)$ \\
Coordenação do Psiquiatra/Técnico de & 3 & $0,49(0,25-0,67)$ \\
Referência & & $0,37(0,10-0,58)$ \\
Coordenação Geral & 1 & $0,05(-0,22-0,32)$ \\
Médico Clínico & 2 & $0,00(-0,27-0,27)$ \\
Transição Internação-Ambulatório & 5 & $0,00(-0,95-0,95)$ \\
Transição do Serviço de Emergência & 4 & SR \\
Mudança de Psiquiatra & 4 & $0,41(-0,60-0,91)$ \\
Mudança de Técnico de Referência & 4 & SR \\
Mudança de Domicílio & 5 & $0,00(-0,99-0,99)$ \\
Escala Completa & 59 & $0,77(0,63-0,86)$ \\
\hline
\end{tabular}

SR: sem resposta - Não foi possível calcular o ICC.

A consistência interna pelo alfa de Cronbach apresentou-se alta para três subescalas, moderada para duas subescalas e baixa para uma subescala, tanto na primeira (teste) quanto na segunda (reteste) aplicação da escala (Tabela 2).
Para duas subescalas o alfa de Cronbach apresentou diferenças entre o teste e o reteste, porém as variações em números demonstraram-se razoavelmente pequenas.

Não foi possível calcular o alfa de Cronbach para as subescalas transição internação-ambulatório, para o teste, e transição do serviço de emergência, para o reteste, possivelmente pela não variabilidade entre as respostas. Para a subescala mudança de domicílio, o alfa de Cronbach apresentou valor igual a zero $(a=0)$, tanto no teste como no reteste, e para a subescala mudança de técnico de referência, não foi possível calcular o alfa de Cronbach por causa do número muito pequeno de respondentes.

O alfa de Cronbach não foi calculado para a subescala coordenação geral, por se tratar de um indicador com um único item, e para a subescala médico clínico, pelo número reduzido de itens.

\section{DISCUSSÃO}

O processo de tradução e retrotradução da CONNECT se mostrou adequado tanto em relação à avaliação da equivalência conceitual e de itens quanto à avaliação da equivalência semântica, e o formato e o modo de aplicação da escala também se mostraram satisfatórios, sendo mantidos os mesmos itens e as mesmas opções de respostas da escala original.

Tabela 2. Escore médio, DP e consistência interna pelo alfa de Cronbach por subescala, para todos os itens da CONNECT

\begin{tabular}{|c|c|c|c|c|c|c|c|}
\hline \multirow[b]{2}{*}{ Subescalas do CONNECT } & \multirow[b]{2}{*}{$N^{\circ}$ de itens } & \multicolumn{3}{|c|}{ Teste } & \multicolumn{3}{|c|}{ Reteste } \\
\hline & & $\begin{array}{l}\text { Escore } \\
\text { Médio }\end{array}$ & DP & $\begin{array}{l}\text { Alpha de } \\
\text { Cronbach }\end{array}$ & $\begin{array}{l}\text { Escore } \\
\text { Médio }\end{array}$ & DP & $\begin{array}{l}\text { Alphade } \\
\text { Cronbach }\end{array}$ \\
\hline Conhecimento do Psiquiatra & 6 & 17,54 & 6,65 & 0,84 & 16,10 & 6,36 & 0.82 \\
\hline Conhecimento do Técnico de Referência & 6 & 16,74 & 7,34 & 0,85 & 17,18 & 7,54 & 0,89 \\
\hline Disponibilidade do Psiquiatra & 3 & 5,82 & 2,89 & 0,53 & 5,94 & 3,18 & 0,61 \\
\hline Disponibilidade do Técnico de Referência & 3 & 7,36 & 3,62 & 0,66 & 6,84 & 3,48 & 0,58 \\
\hline Psiquiatra/Técnico de Referência/Profissional do CAPS & 7 & 13,66 & 3,88 & 0,36 & 13,90 & 4,37 & 0,52 \\
\hline Flexibilidade do Psiquiatra/Técnico de Referência & 6 & 20,86 & 6,45 & 0,69 & 21,68 & 6,85 & 0,74 \\
\hline Coordenação do Psiquiatra/Técnico de Referência & 3 & 11,24 & 3,59 & 0,60 & 10,26 & 4,40 & 0,71 \\
\hline Coordenação Geral & 1 & 4,52 & 0,97 & -- & 4,68 & 0,81 & -- \\
\hline Médico Clínico & 2 & 2,00 & 0,00 & -- & 2,16 & 1,13 & -- \\
\hline Transição Internação-Ambulatório & 5 & 22,87 & 2,10 & SR & 24,00 & 2,00 & 0,00 \\
\hline Transição do Serviço de Emergência & 4 & 15,33 & 6,40 & 0,88 & 18,16 & 1,60 & SR \\
\hline Mudança de Psiquiatra & 4 & 12,18 & 6,72 & 0,88 & 17,00 & 5,62 & 0,90 \\
\hline Mudança de Técnico de Referência & 4 & 4,00 & $S R$ & $S R$ & 16,00 & $S R$ & SR \\
\hline Mudança de Domicílio & 5 & 6,66 & 1,96 & 0,00 & 9,00 & 0,00 & 0,00 \\
\hline Escala Completa & 59 & 108,80 & 27,79 & 0,61 & 105,74 & 28,32 & 0,63 \\
\hline
\end{tabular}

SR: sem resposta - Não foi possível calcular o escore médio e o Alpha de Cronbach.

DP: desvio-padrão. 
Em relação à população de estudo, as amostras não se diferenciaram em relação às características sociodemográficas e categorias diagnósticas.

Em relação à confiabilidade temporal ou intraobservador pelo método estatístico kappa ponderado e PABAK, alguns itens apresentaram pequena ou pobre confiabilidade pelo kappa ponderado. Contudo, quando ajustados pela prevalência, aumentaram consideravelmente, mostrando-se adequado o seu uso para evitar possíveis distorções nos resultados. Dessa forma, 34 itens apresentaram confiabilidade de moderada à quase perfeita, demonstrando estabilidade entre as respostas. E o percentual de concordância, quando analisado isoladamente para cada item, também demonstrou reprodutibilidade satisfatória.

A análise item a item pelo kappa ponderado e PABAK não foi realizada nos estudos de confiabilidade da CONNECT para a língua inglesa e a língua espanhola, não podendo, com isso, haver comparação dos dados encontrados pelo presente estudo.

Os resultados da análise da concordância pelo ICC diferiram dos encontrados no estudo original em inglês ${ }^{3}$ e na versão em espanhol ${ }^{4}$. Quanto ao resultado para a subescala médico clínico, ela apresentou ICC igual a zero, porém o percentual de concordância para essa subescala foi de 98\%. Para os dois itens dessa subescala, não houve variabilidade entre as respostas. Essa homogeneidade dos dados pode ser uma das razões para o ICC baixo, visto que, quando as medidas são homogêneas, o ICC pode apresentar escore menor em virtude da ausência de diferença estatística verificada na análise de variância dos dados ${ }^{17}$.

Outro ponto importante a ser levantado quanto a esse resultado é o fato de a maioria dos pacientes ter respondido negativamente a essas perguntas. Uma provável explicação seria a de que os participantes responderam que o psiquiatra e o técnico de referência não tinham contato com seu médico clínico por não possuírem um médico clínico que os acompanhassem continuamente.

Ainda em relação à subescala médico clínico, é importante destacar que no estudo de adaptação da CONNECT para o espanhol ${ }^{4}$ essa subescala foi excluída. Os autores encontraram em sua amostra muitos pacientes que não eram tratados especificadamente por psiquiatras, e sim por médicos clínicos, e entenderam que essa também poderia ser a realidade de outros locais que prestam cuidados de saúde a pacientes com transtorno mental.

Sobre o resultado para o indicador de um único item, as respostas também variaram pouco, 32 pacientes responderam achar seu tratamento de saúde mental bem organizado, tanto na primeira como na segunda aplicação da escala. Alguns autores ${ }^{18}$ destacam que o paciente com transtorno mental tem conhecimento único da vivência de sua doença, sintomas, necessidades e experiência com os serviços de saúde mental, sendo essa perspectiva diferente da visão dos profissionais. E, ainda, que a falta de insight e os sintomas po- sitivos e negativos da doença podem resultar em dificuldade de aceitação da doença e percepção do tratamento ${ }^{19}$.

Em relação às subescalas de transição de serviços, os pacientes que apresentaram um episódio agudo recente e precisaram ser atendidos no serviço de emergência e/ou de internação podem ter experimentado sintomas mais graves da doença e, com isso, apresentarem maior comprometimento cognitivo ou de memória, fato que poderia influenciar na confiabilidade de suas respostas e justificaria a inconsistência das respostas em relação a essas subescalas.

É importante frisar a natureza qualitativa da escala. A versão original em inglês da CONNECT foi desenvolvida a partir de um estudo etnográfico ${ }^{20}$ e procurou refletir as perspectivas dos usuários do serviço, utilizando a premissa de que eles têm maior competência para definir o que constitui uma saúde mental adequada. A continuidade do cuidado foi descrita por meio das interações diárias de pessoas que fornecem e recebem cuidados. Portanto, as dimensões subjetivamente avaliadas, no que tange à percepção dos usuários em relação ao serviço e sua interação com os fornecedores de cuidado, podem sofrer grandes variações étnicas, culturais e sociais.

Em relação à consistência interna pelo alfa de Cronbach, os valores encontrados para seis subescalas ficaram próximos aos valores encontrados no estudo americano da CONNECT original $^{3}$ e no estudo da versão adaptada para o espanhol ${ }^{4}$

Assim como no estudo de Chavez et al. ${ }^{4}$, a consistência interna para o indicador de um único item "coordenação geral" não foi avaliada. Neste estudo também não foi avaliada a consistência interna para a subescala médico clínico por causa do número reduzido de itens, já que o valor do alfa de Cronbach é influenciado pelo número de itens que compõem uma escala ${ }^{21,22}$.

Em relação aos valores de alfa de Cronbach encontrados para as subescalas de transição, um aspecto importante a ser considerado é o tamanho da amostra. Um número reduzido de pacientes respondeu a essas subescalas, o que pode ter afetado os resultados. Segundo Maroco e Garcia-Marques²1 e Cortina ${ }^{22}$, o valor de alfa pode ser afetado não apenas pela correlação entre as respostas obtidas, mas também pelo número de questões feitas e por redundância.

Outro fator a ser considerado é o próprio modelo estrutural da CONNECT, com várias subescalas independentes, o que poderia também explicar a baixa consistência encontrada por não haver uma única causa comum entre as subescalas ${ }^{23}$.

É importante destacar algumas dificuldades práticas no estudo da confiabilidade teste-reteste, que podem fazer com que os dados não sejam exatamente comparáveis como: fornecimento de respostas iguais pela lembrança das respostas da primeira aplicação do questionário; mudança de respostas na segunda aplicação do questionário por uma maior crítica ao assunto abordado; diminuição do empenho em fornecer respostas mais confiáveis na segunda aplicação 
do questionário; e eventos recentes entre as duas aplicações que possam alterar as respostas ${ }^{24}$.

\section{CONCLUSÕES}

A CONNECT é a primeira medida de continuidade do cuidado adaptada para o contexto cultural brasileiro. Sua adaptação mostrou adequação das equivalências entre a versão original e a versão em português (Brasil), com resultados plenamente satisfatórios.

Os resultados obtidos no estudo da versão original em inglês da CONNECT e na versão em espanhol apontaram para valores adequados de confiabilidade. Esses resultados diferiram dos resultados obtidos neste estudo, principalmente em relação às subescalas de transição, comprometidas por causa do pequeno número de respondentes. Contudo, esses resultados não invalidam a utilização da CONNECT no contexto cultural brasileiro, pois, para as subescalas conhecimento, disponibilidade, flexibilidade e coordenação, os resultados se mostraram bastante satisfatórios.

Vale salientar que este estudo constitui a primeira fase para a obtenção de uma medida de continuidade do cuidado válida para pacientes brasileiros com transtornos mentais graves. Estudos adicionais para examinar a validade da CONNECT ainda se fazem necessários.

Assim, acreditamos que este estudo pode contribuir para que outros possam ser desenvolvidos focando especificamente a continuidade do cuidado em pacientes com transtornos mentais graves, tema ainda pouco estudado no país, mas de grande importância no momento para a área de saúde mental. Este estudo também pode estimular a utilização de escalas confiáveis e válidas que possam não somente mensurar a continuidade do cuidado, mas também direcionar as ações e práticas de cuidado em saúde mental.

\section{CONTRIBUIÇÕES INDIVIDUAIS}

Michelli Melo Grama - Pesquisadora principal do estudo, participou de todas as etapas na elaboração do artigo.

Lúcia Abelha - Orientou em todas as etapas do artigo, incluindo a revisão.

Giovanni Lovisi - Coorientou em todas as etapas do artigo, incluindo a revisão.

Diego de Lima Fonseca - Colaborou na coleta de dados, análise estatística, descrição dos resultados e na revisão do artigo.

\section{CONFLITO DE INTERESSE}

Nada a declarar.

\section{REFERÊNCIAS}

1. Amarante P. Saúde mental e atenção Psicossocial. Rio de Janeiro: Fiocruz; 2007. 120p.

2. Bachrach LL. Continuity of care for chronic mental patients: a conceptual analysis. Am J Psychiatry. 1981;138(11):1449-56.

3. Ware NC, Dickey B, Tugenberg T, McHorney CA. CONNECT: a measure of continuity of care in mental health services. Ment Health Serv Res. 2003;5(4):209-21.

4. Chavez LM, Canino G, Shrout PE, Barrio C, Ware NC. Psychometric evaluation of the Spanish version of CONNECT: a measure of continuity of care in mental health services. Int J Methods Psychiatr Res. 2007;16(1):23-33.

5. Bandeira M, Gelinas D, Lesage A. Desinstitucionalização: o programa de acompanhamento intensivo na comunidade. J Bras Psiquiatr. 1998:47(12):627-40.

6. World Health Organization. Process of translation and adaptation of instruments. Disponivel em: <http://www.who.int/substance_abuse/research_tools/translation/en/>. Acesso em: 6 ago. 2012

7. Herdman M, Fox-Rushby J, Badia X. A model of equivalence in the cultural adaptation of HRQoL instruments: the universalist approach. Qual Life Res. 1998;7(4):323-35.

8. Reichenheim ME, Moraes CL. Operacionalização de adaptação transcultural de instrumentos de aferição usados em epidemiologia. Rev Saúde Pública. 2007;41(4):1-9.

9. Streiner DL, Norman GR. Health Measurement Scales: a practical guide to their development and use. 2nd ed. London: Oxford University Press; 1995.

10. Moraes $(L$, Hasselman MH, Reichenheim ME. Adaptação transcultural para o português do instrumento "Revised Conflict Tatics Scales (CTS2)" utilizado para identificar violência entre casais. Cad Saude Publica. 2002:18(1):163-76.

11. Organização Mundial da Saúde. CID-10 - Classificação Estatística Internacional de Doenças e Problemas Relacionados à Saúde. 10ª ed. São Paulo: Universidade de São Paulo. v. I.

12. Bandeira M, Lima LA, Gonçalves S. Qualidades psicométricas no papel da Escala de Habilidades de Vida Independente de pacientes psiquiátricos (ILSS-BR). Rev Psiquiatr Clín. 2003;30(4):121-5.

13. Schlithler AC, Scazufca M, Busatto G, Coutinho LM, Menezes PR. Reliability of the Brazilian version of the Camberwell Assessment of Needs (CAN) in first-episode psychosis cases in São Paulo, Brazil. Rev Bras Psiquiatr. 2007;29(2):160-3.

14. Sim J, Wright CC. The kappa statistic in reliability studies: use, interpretation, and sample size requirements. Phys Ther. 2005;85(3):257-68.

15. Mcdowell I. Measuring health: a guide to rating scales and questionnaires. 3rd ed. New York: Oxford University Press; 2006

16. Freitas ALP, Rodrigues SG. A avaliação da confiabilidade de questionários: uma análise utilizando o coeficiente alfa de Cronbach. In: Anais do Simpósio de Engenharia de Produção 8; 2005; Bauru, SP. Bauru: Unesp; 2005. Disponível em: <http://www.simpep.feb.Unesp.br/ anais/anais >. Acesso em: 6 nov. 2013.

17. Portney $L G$, Watkins MP. Reliability. In: Portney LG, Watkins MP. Foundations of clinical research: application to practice. 2nd ed. New Jersey: Prentice Hall Health; 2000.

18. Tait $\mathrm{L}$, Lester $\mathrm{H}$. Encouraging user involvement in mental health services. Adv Psychiatr Treat. 2005;11:168-75.

19. Hunter R, Cameron R. The Scottish Schizophrenia Outcomes Study: a new paradigm for utilizing self-report assessments from patients with schizophrenia. Eur Psychiatr Rev. 2008; $1(2): 7-9$

20. Ware NC, Tugenberg T, Dickey B, McHorney CA. An ethnographic study of the meaning of continuity of care in mental health services. Psychiatr Serv. 1999;50(3):395-400.

21. Maroco J, Garcia-Marques T. Qual a fiabilidade do alfa de Cronbach? Questões antigas e soluções modernas? Laboratório de Psicologia. 2006;4(1):65-90. Disponível em: <http:// repositorio.ispa.pt/handle/10400.12/133>. Acesso em: 3 fev. 2014.

22. Cortina JM. What is coefficient alpha? An examination of theory and applications. J Appl Psychol. 1993;78(1):98-104.

23. Edwards JR, Bagozzi RP. On the nature and direction of relationships between constructs and measures. Psychol Methods. 2000;5(2):155-74.

24. Moser CA, Kalton G. Scaling methods. In: Moser CA, Kalton G. Survey Methods in social investigation. 2nd ed. London: Heinemam; 1975. 


\section{Anexo 1}

CONNECT: uma medida de continuidade do cuidado

Agora eu quero perguntar algumas coisas sobre o cuidado que você recebeu neste local. Por favor, me aponte qual das opções a seguir melhor reflete a sua opinião ou o que você sente.

\begin{tabular}{|c|c|}
\hline ESCALAS & ITENS \\
\hline CONHECIMENTO DO MÉDICO & $\begin{array}{l}\text { 1. Quanto (nome do psiquiatra) sabe sobre seus pontos fortes? } \\
\text { 2. Quanto (nome do psiquiatra) sabe sobre os relacionamentos que são importantes para você? } \\
\text { 3. Quanto (nome do psiquiatra) sabe sobre sua história familiar? } \\
\text { 4. Quanto (nome do psiquiatra) sabe sobre sua relação com sua família? } \\
\text { 5. Quanto (nome do psiquiatra) sabe sobre o que perturba você? } \\
\text { 6. Quanto (nome do psiquiatra) sabe sobre as situaçõos que são estressantes para você? }\end{array}$ \\
\hline \multicolumn{2}{|r|}{ 1-nada, 2 - um pouco, 3 - algumas coisas, 4 - bastante, 5 - tudo } \\
\hline CONHECIMENTO DO MÉDICO RESPONSÁVEL/TERAPEUTA & $\begin{array}{l}\text { 1. Quanto }(M R / T) \text { sabe sobre seus pontos fortes? } \\
\text { 2. Quanto }(M R / T) \text { sabe sobre seus objetivos pessoais? } \\
\text { 3. Quanto }(M R / T) \text { sabe do que você gosta? } \\
\text { 4. Quanto }(M R / T) \text { sabe sobre o que perturba você? } \\
\text { 5. Quanto }(M R / T) \text { sabe do que você tem medo? } \\
\text { 6. Quanto }(M R / T) \text { sabe sobre em quem você pode confiar? }\end{array}$ \\
\hline \multicolumn{2}{|r|}{ 1-nada, 2 - um pouco, 3 - algumas coisas, 4 - bastante, 5 - tudo } \\
\hline DISPONIBILIDADE DO PSIQUIATRA & $\begin{array}{l}\text { No último ano, } \\
\text { 1. Eu deixei uma mensagem no telefone para o ( } p s q) \text {. } \\
\text { 2. Eu falei com o (psq) por telefone no dia de plantão. } \\
\text { 3. } 0 \text { (psq) retornou minhas ligações no mesmo dia. }\end{array}$ \\
\hline \multicolumn{2}{|r|}{1 - nunca, 2 -raramente, 3 -às vezes, 4 - regularmente, 5 - sempre } \\
\hline DISPONIBILIDADE DO MÉDICO RESPONSÁVEL/TERAPEUTA & $\begin{array}{l}\text { No último ano, } \\
\text { 1. Eu deixei uma mensagem no telefone para o (psq). } \\
\text { 2. Eu falei com o (psq) por telefone no dia de plantão. } \\
\text { 3. } 0 \text { (MR/T) retornou minhas ligações no mesmo dia. }\end{array}$ \\
\hline \multicolumn{2}{|r|}{1 - nunca, 2 - raramente, 3 -às vezes, 4 - regularmente, 5 - sempre } \\
\hline SUPORTE CLÍNICO & $\begin{array}{l}\text { No último ano, um de meus acompanhantes... } \\
\text { 1. Me deu uma carona a algum lugar. } \\
\text { 2. Me levou para fazer compras. } \\
\text { 3. Me comprou uma xícara de café. } \\
\text { 4. Me ajudou com a minha dieta. } \\
\text { 5. Me ajudou a preencher formulários/documentos. } \\
\text { 6. Me ajudou a seguir cuidados de saúde. }\end{array}$ \\
\hline \multicolumn{2}{|r|}{1 - nunca, 2 - raramente, 3 - às vezes, 4 - regularmente, 5 - sempre } \\
\hline FLEXIBILIDADE DO MÉDICO & $\begin{array}{l}\text { 1. (MR/T) parará o que está fazendo para me ajudar. } \\
\text { 2. Eu posso remarcar consultas com ele (MR/T). } \\
\text { 3. Eu posso marcar consultas com (MR/T) em cima da hora. } \\
\text { 4. (psq) parará o que está fazendo para me ajudar. } \\
\text { 5. Eu posso remarcar consultas com ele (psiq). } \\
\text { 6. Eu posso marcar consultas com (psiq) em cima da hora. }\end{array}$ \\
\hline \multicolumn{2}{|r|}{1 - nunca, 2 - raramente, 3 -às vezes, 4 - regularmente, 5 - sempre } \\
\hline COORDENAÇÃO DO CLÍNICO & $\begin{array}{l}\text { 1. Com qual frequência o (psiq) e (MR/T) falam entre si a respeito do seu tratamento? } \\
\text { 2. Com qual frequência o (psiq) e (MR/T) trabalham juntos como uma equipe? } \\
\text { 3. Com qual frequência o (psiq) e (MR/T) concordam sobre seu tratamento? }\end{array}$ \\
\hline COORDENAÇÃO GERAL & No geral, seu tratamento de saúde mental é bem organizado? \\
\hline \multicolumn{2}{|r|}{1 - nunca, 2 - raramente, 3 -às vezes, 4 - regularmente, 5 - sempre } \\
\hline CUIDADOS PRIMÁRIOS & $\begin{array}{l}\text { 1. Com qual frequência (psiq) tem contato com seu clínico? } \\
\text { 2. Com qual frequência (MR/T) tem contato com seu clínico? }\end{array}$ \\
\hline \multicolumn{2}{|r|}{1 - nunca, 2 -raramente, 3 -às vezes, 4 - regularmente, 5 - sempre } \\
\hline TRANSIÇÃO INTERNAÇÃOO-AMBULATÓRIO & $\begin{array}{l}\text { 1. Eu tive um bom planejamento de alta. } \\
\text { 2. Eu, em conjunto com o pessoal do hospital, concordei com o planejamento de alta. } \\
\text { 3. Eu recebi a medicação para tomar em casa. } \\
\text { 4. Eu tive agendado o meu acompanhamento no ambulatório. } \\
\text { 5. No geral, a transição entre a internação e o tratamento ambulatorial foi tranquila. }\end{array}$ \\
\hline
\end{tabular}


1-discordo plenamente, 2-discordo em parte, 3 - nem discordo nem concordo, 4- concordo, 5 - concordo plenamente

\begin{tabular}{|c|c|}
\hline TRANSIÇÃO DO SERVIÇO DE EMERGÊNCIA & $\begin{array}{l}\text { 1. Fui atendido em menos de } 2 \text { horas. } \\
\text { 2. Fui atendido rapidamente. } \\
\text { 3. Eu pude opinar nas decisões sobre o tratamento. } \\
\text { 4. } 0 \text { processo foi tranquilo. }\end{array}$ \\
\hline \multicolumn{2}{|c|}{ 1- discordo plenamente, 2 - discordo em parte, 3 - nem discordo nem concordo, 4- concordo, 5 - concordo plenamente } \\
\hline MUDANÇA DE PSIQUIATRA & $\begin{array}{l}\text { Tendo como base sua mais recente mudança de psiquiatra, quando seu antigo psiquiatra... } \\
\text { 1. Ajudou, por informar com antecedência a sua saída? } \\
\text { 2. Te ajudou, preparando você para a mudança? } \\
\text { 3. Conversou com você sobre o que você sentia sobre essa mudança? } \\
\text { 4. No geral, fez uma transição tranquila? }\end{array}$ \\
\hline \multicolumn{2}{|r|}{ 1- nunca, 2 - um pouco, 3 - algumas vezes, 4 - muito, 5 - sempre } \\
\hline MUDANÇA DE GERENTE DE CASO/TERAPEUTA & $\begin{array}{l}\text { Pesando sobre sua recente mudança de terapeuta ou gerente, quanto é que seu antigo (MR/T)... } \\
\text { 1. Ajudou, por informar com antecedência a sua saída? } \\
\text { 2. Te ajudou, preparando você para a mudança? } \\
\text { 3. Conversou com você sobre o que você sentia sobre essa mudança? } \\
\text { 4. No geral, fez uma transição tranquila? }\end{array}$ \\
\hline \multicolumn{2}{|r|}{ 1- nunca, 2 - um pouco, 3 - algumas vezes, 4 - muito, 5 - sempre } \\
\hline MUDANÇA DE DOMICÍLIO & $\begin{array}{l}\text { Tendo como base sua mais recente mudança de casa, quanto os cuidadores ajudaram você... } \\
\text { 1. A encontrar um lar? } \\
\text { 2. A planejar uma visita antes da sua mudança? } \\
\text { 3. A transportar seus pertences? } \\
\text { 4. A te deixar bem acomodado? } \\
\text { 5. A deixar o processo de transição mais tranquilo? }\end{array}$ \\
\hline & -nunca, 2 - um pouco, 3 - algumas vezes, 4 - muito, 5 - sempre \\
\hline
\end{tabular}

